University of Wollongong

Research Online

Faculty of Engineering and Information

Faculty of Engineering and Information

Sciences - Papers: Part B

Sciences

2017

\title{
A Potential field approach-based trajectory control for autonomous electric vehicles with in-wheel motors
}

Boyuan $\mathrm{Li}$

University of Wollongong, bl995@uowmail.edu.au

Haiping Du

University of Wollongong, hdu@uow.edu.au

Weihua Li

University of Wollongong, weihuali@uow.edu.au

Follow this and additional works at: https://ro.uow.edu.au/eispapers1

Part of the Engineering Commons, and the Science and Technology Studies Commons

Research Online is the open access institutional repository for the University of Wollongong. For further information contact the UOW Library: research-pubs@uow.edu.au 


\title{
A Potential field approach-based trajectory control for autonomous electric vehicles with in-wheel motors
}

\author{
Abstract \\ The studies on the autonomous electric vehicle are quite attractive due to fewer human-induced errors \\ and improved safety in recent years. Extensive research has been done on the autonomous steering \\ control of the mobile robot, but study on the on-road autonomous electric vehicle is still limited. This \\ paper proposes a potential field method to achieve the trajectory control of the autonomous electric \\ vehicle with in-wheel motors. Instead of strictly following a desired path, this method can form a steering \\ corridor with a desired tracking error tolerance and the vehicle can be steered smoothly with less control \\ effort. In this paper, the innovative potential filed function is presented first to determine the desired \\ vehicle yaw angle. Then, according to this desired yaw angle, a two-level trajectory controller is proposed \\ to achieve the trajectory control. Simulation results are shown to prove that this suggested trajectory \\ controller can successfully control the vehicle to move within the desired road boundary and improve the \\ handling and stability performance of the vehicle.

\section{Disciplines} \\ Engineering | Science and Technology Studies

\section{Publication Details} \\ B. Li, H. Du \& W. Li, "A Potential field approach-based trajectory control for autonomous electric vehicles \\ with in-wheel motors," IEEE Transactions on Intelligent Transportation Systems, vol. 18, (8) pp. 2044-2055, \\ 2017.
}




\title{
A Potential Field Approach Based Trajectory Control for Autonomous Electric Vehicles with In-wheel Motors
}

\author{
Boyuan Li, Haiping Du, Member, IEEE and Weihua Li, Member, IEEE
}

\begin{abstract}
The studies on the autonomous electric vehicle are quite attractive due to the less human induced error and the improved safety in recent years. Extensive research has been done on the autonomous steering control of the mobile robot, but the study on the on-road autonomous electric vehicle is still limited. This study proposes a potential field method to achieve the trajectory control of the autonomous electric vehicle with in-wheel motors. Instead of strictly following a desired path, this method can form a steering corridor with a desired tracking error tolerance and the vehicle can be steered smoothly with smaller control effort. In this paper, the innovative potential filed function is presented first to determine the desired vehicle yaw angle. Then according to this desired yaw angle, a two-level trajectory controller is proposed to achieve the trajectory control. Simulation results are shown to prove that this suggested trajectory controller can successfully control the vehicle to move within the desired road boundary and improve the handling and stability performance of the vehicle.
\end{abstract}

Index Terms-Autonomous vehicle control, vehicle dynamics, potential field method, four-wheel independent steering, four-wheel independent driving.

\begin{tabular}{ll}
$m$ & \multicolumn{1}{c}{ NOMENCLATURE } \\
$I_{z}$ & Vehicle mass \\
$l_{f}$ & Front wheel base \\
$l_{r}$ & Rear wheel base \\
$b_{f}$ & Front track width \\
$b_{r}$ & Rear track width \\
$F_{x i}$ & Longitudinal tyre force of individual wheel \\
$F_{y i}$ & Lateral tyre force of individual wheel \\
$F_{s i}$ & Tyre side force of individual wheel \\
$F_{t i}$ & Tyre traction or brake force of individual wheel \\
$\delta_{i}$ & Steering angle of individual wheel \\
$F_{z i}$ & Vertical load of individual wheel
\end{tabular}

This paragraph of the first footnote will contain the date on which you submitted your paper for review. This work was supported in part by Australian Research Council Discovery Project under Grant DP140100303.

B. $\mathrm{Li}$ is with the School of Electrical, Computer and Telecommunications Engineering, University of Wollongong, Wollongong, NSW 2522, Australia (e-mail: bl995@uowmail.edu.au).

H. Du is with the School of Electrical, Computer and Telecommunications Engineering, University of Wollongong, Wollongong, NSW 2522, Australia (e-mail: hdu@uow.edu.au).

W. Li is with the School of Mechanical, Material and Mechatronic Engineering, University of Wollongong, Wollongong, NSW 2522, Australia (e-mail:Weihuali@uow.edu.au) $u_{i} \quad$ Vehicle velocity component in the wheel plane of individual wheel

$\alpha_{i} \quad$ Lateral side-slip angle of individual wheel

$s_{i} \quad$ Longitudinal slip ratio of individual wheel

$T_{i} \quad$ Traction or brake torque of individual wheel

$\omega_{i} \quad$ Wheel angular velocity of individual wheel

$i \quad$ Corresponding to the front left, front right, rear left and rear right wheel $(=f l, f r, r l, r r)$

$X \quad$ Vehicle longitudinal position in the global coordinate system

$Y \quad$ Vehicle lateral position in the global coordinate system

$Y_{u b} \quad$ Lateral position of the upper boundary in the global coordinate system

$Y_{l b} \quad$ Lateral position of the lower boundary in the global coordinate system

$v_{x} \quad$ Longitudinal velocity of vehicle

$v_{x d} \quad$ Desired longitudinal velocity of vehicle

$\tilde{v}_{x} \quad$ Longitudinal velocity error in the global coordinate system

$v_{y} \quad$ Lateral velocity of vehicle

$v_{y d-g}$ Desired vehicle lateral velocity of the central line of the desired trajectory in the global coordinate system

$r \quad$ Yaw rate of the vehicle

$\varphi \quad$ Yaw angle of the vehicle

$\tilde{\varphi} \quad$ Yaw angle error

$\varphi_{d} \quad$ Desired yaw angle

$\varphi_{\text {act }}$ Vehicle actual yaw angle

$g \quad$ Acceleration gravity

$h \quad$ Height of vehicle centre of gravity above the ground

$\mu \quad$ Tyre-road friction coefficient

$C_{\alpha} \quad$ Tyre lateral cornering stiffness

$C_{s} \quad$ Tyre longitudinal cornering stiffness

$\varepsilon_{r} \quad$ Constant value in Dugoff tyre model

$R_{\omega} \quad$ Vehicle wheel radius

$I_{\omega} \quad$ Wheel moment of inertial

$U_{\text {att }} \quad$ Attractive potential

$U_{\text {rep }} \quad$ Repulsive potential

$U_{s} \quad$ Potential function that minimize the yaw angle rate

$\alpha_{0} \quad$ Scaling factor of optimization problem

$\alpha_{v} \quad$ Scaling factor of optimization problem

$b_{1} \quad$ Scaling factor of optimization problem

$b_{2} \quad$ Scaling factor of optimization problem

$c \quad$ Scaling factor of optimization problem

$\tilde{e}_{y} \quad$ Lateral position error in the global coordinate system

$M_{z} \quad$ Vehicle yaw moment 


\section{INTRODUCTION}

$\mathrm{N}$ owadays, intensive research has been done into autonomous vehicles [1-4]. The ultimate goal of automated driving is to reduce accidents caused by human error and improve safety. In addition, full automation can significantly improve the road capacity and diminish air pollution because of a more efficient use of fuel [5]. For the autonomous vehicle, the human controlled steering system is replaced by the autonomous steering control system and consequently the automated steering control is the core part of the autonomous vehicle system.

In the area of advanced robotics, the desired trajectory is determined at first and the steering system of the robotic is controlled so that the robot will follow the desired trajectory exactly at every time step [6][7]. For the robotic control, this steering control approach is reasonable since the robotic has high mobility and the moving velocity is relatively low in the in-door condition. For the steering control of on-road vehicle, however, this kind of approaches need large amount of control effort and the actual steering angle will oscillate abruptly due to the limitation of the vehicle mobility. The other control performance such as smoothness of the vehicle motion would also be hard to achieve.

In addition to the direct trajectory tracking method, the potential field method is also quite attracting in the research area of the autonomous steering control of mobile robotic. The steering control method based on the potential fields can form a steering corridor with a desired tracking error tolerance and the vehicle can be steered smoothly with smaller control effort compared with the direct trajectory tracking method. The total potential normally includes the attractive potential to reach the desired position and repulsive potential to avoid the obstacle. Jaradat et al. utilised the fuzzy model and TSK model to develop the total attractive and repulsive potential force applied on the autonomous mobile robot [8]. Khatib presented an unique real-time obstacle avoidance approach for the mobile robot based on the artificial potential field method [9]. In addition, Pan et al. used the fuzzy controller to improve the artificial potential field method and safeguarded the reliability of the path planning and path smoothness [10]. Ge and Cui proposed the dynamic motion planning method for the mobile robot where the target and obstacle are moving by using the potential field method [11].

The potential field method is advantageous to control the vehicle to follow a more smooth trajectory and to decrease the total control effect compared with the strictly trajectory following method. Thus, the potential field method is quite attractive for the autonomous control of the on-road electric vehicle with limited mobility and high velocity. The above papers about the potential field method, however, are mainly focused on the mobile robot and the virtual longitudinal and lateral control forces are required to achieve the control trajectory. In the area of the on-road vehicle, the longitudinal motion, lateral motion and yaw motion are highly coupled and only tracking the virtual forces can hardly achieve the desired trajectory.

One possible way to control the trajectory of the on-road vehicle is to achieve the desired yaw angle instead of the tracking of the virtual forces or the desired position. Park and Gerdes proposed the trajectory control method by tracking the desired yaw angle based on the on-road vehicle dynamics model. Then, according to the desired vehicle motion, the actual actuators are allocated by equally using the friction capability of each tyre [12]. However, the yaw angle depends on time, and the desired road trajectory and road boundary depend on positions. In order to achieve the desired trajectory by using the yaw angle control, the time-dependent real-time vehicle states should be transferred into the position-dependent desired trajectory and road boundary.

For traditional internal combust engine (ICE) vehicles, only one steering control input and one driving input are used to achieve multiple control targets, and this limits control performance. With the development of the innovative technology of electric vehicles with in-wheel steering and driving motors, four-wheel independent steering (4WIS) and four-wheel independent driving (4WID) can be achieved [13][14]. 4WIS-4WID vehicles have the advantages that the number of the control actuators is 8 (four steering control actuators and four driving control actuators). The large number of control actuators can achieve the redundant control, which means the number of the control actuators is larger than the number of control targets and additional control targets can be achieved. In addition, more control inputs are available for the longitudinal and lateral motion control and the total control workload can be minimised [15][16]. In the yaw angle based trajectory control for the on-road vehicle, the optimisation of the control workload of actuators is advantageous for the vehicle overall handling and stability performance.

In this study, an innovative potential field method aiming to achieve the vehicle trajectory control based on yaw angle control is proposed. This potential filed method includes the attractive potential function, repulsive potential function and the yaw angle potential function that minimises the yaw angle change rate. Instead of using the relative positions, this paper uses the difference between the desired velocity and the actual velocity to describe the attractive potential function since the vehicle lateral velocity is directly related to the yaw angle. The repulsive potential function is proposed to guarantee the actual vehicle trajectory is constrained by the upper and lower boundary. The vehicle dynamics motion is described by the time-derivative equations and these equations should be transferred into the position-dependent equations that describe the vehicle actual trajectory to guarantee the satisfaction of the road boundary. In addition, the yaw angle potential function is suggested in this paper to minimise the yaw angle change rate and improve the handling and stability of the vehicle. These potential functions can be minimised to determine the real-time desired yaw angle and longitudinal velocity. Then according to the desired yaw angle and longitudinal velocity, a two-level vehicle trajectory controller is suggested to track this desired yaw angle and the desired trajectory. In the upper level, the desired vehicle total longitudinal force, total lateral force and yaw moment are determined according to the desired yaw angle and desired longitudinal velocity. In the lower level, the 
controlled values of individual steering and driving actuator are optimally allocated in the 4WIS-4WID electric vehicle to achieve the desired total longitudinal force, lateral force and yaw moment.

It is noted that in the literature, the eco-driving method is extensively studied to minimise the fuel use of the ICE vehicle or the electric power output of the electric vehicle. The target of eco-driving is mainly achieved by developing an optimal driving strategy subjected to the surrounding condition of the vehicle and traffic flow. Bath and Boriboonsomsin proposed the dynamic eco-driving strategy to provide the driver the advised speed [17]. This eco-diving strategy took advantages of real-time traffic sensing and telematics and included traffic management system to monitor traffic speed, density and flow. Sabbbohi and Farzaneh also developed an optimal driving strategy and control approach to achieve the eco-driving [18]. The control objective is to minimise the fuel consumption and $\mathrm{CO}_{2}$ emission, and not only the vehicle speed but also the engine gear ratio are considered as the control variables. The above studies about vehicle eco-driving focused on minimising the fuel consumption by providing the desired speed or gear ratio and have shown great improvement in the energy-efficiency, but the actual vehicle motion control for the autonomous vehicle to achieve these optimisation targets is less focused. The proposed potential field method in this study, however, can minimise the yaw rate and smooth the actual vehicle trajectory for autonomous vehicle. In addition, the vehicle motion controller based on the 4WIS-4WID vehicle model is designed to achieve this smooth vehicle trajectory.

The main contributions of this study can be summarised as follows: 1) an innovative yaw-angle based potential field function is proposed to achieve the desired road trajectory within certain road boundary and minimise the yaw angle change rate. 2) a two-level vehicle dynamics trajectory controller is proposed to optimally distribute the individual control actuator.

The structure of this paper is organised as follows: first, the four-wheel vehicle dynamics model is introduced and described in Section 2. Then, the proposed potential field method is suggested in Section 3. The upper level controller and the lower level controller of the suggested vehicle trajectory controller are presented in Section 4 and Section 5, respectively. After that, simulations are carried out to verify the effectiveness of the innovative controller in Section 6. Section 7 concludes the paper.

\section{VeHICLE DYNAMICS MODEL}

In this paper, a 4WIS/4WID vehicle model is utilised to describe the dynamics motion of the electric vehicle with in-wheel steering and driving motors [19][20]. This model simulates the conditions of a real vehicle, and is used to validate the performance of the proposed trajectory control method.

The equations of motion of this model are described as follows:

Longitudinal motion:

$$
m \dot{v}_{x}=m v_{y} r+\left(F_{x f l}+F_{x f r}+F_{x r l}+F_{x r r}\right)
$$

Lateral motion:

$$
m \dot{v}_{y}=-m v_{x} r+\left(F_{y f l}+F_{y f r}+F_{y r l}+F_{y r r}\right)
$$

Yaw motion:

$$
\begin{gathered}
I_{z} \dot{r}=l_{f}\left(F_{y f l}+F_{y f r}\right)-l_{r}\left(F_{y r l}+F_{y r r}\right)+\frac{b_{f}}{2}\left(F_{x f l}-F_{x f r}\right) \\
+\frac{b_{r}}{2}\left(F_{x r l}-F_{x r r}\right)
\end{gathered}
$$

The tyre traction or brake force and side force are defined as $F_{t i}$ and $F_{s i}$, respectively, which can be related to the longitudinal and the lateral tyre forces by the steering angle $\delta_{i}$ as follows:

$$
\begin{aligned}
& F_{x i}=F_{t i} \cos \delta_{i}-F_{s i} \sin \delta_{i} \\
& F_{y i}=F_{t i} \sin \delta_{i}+F_{s i} \cos \delta_{i}
\end{aligned}
$$

where $i=f l, f r, r l, r r$, which represents the front left, front right, rear left and rear right wheel, respectively.

$F_{z i}$ is the vertical load of each wheel, which can be calculated as follows [21]:

$$
\begin{aligned}
& F_{z f l}=\frac{m}{l_{f}+l_{r}}\left(\frac{1}{2} g l_{r}-\frac{1}{2} \dot{v}_{x} h-\frac{l_{r}}{b_{f}} \dot{v}_{y} h\right) \\
& F_{z f r}=\frac{m}{l_{f}+l_{r}}\left(\frac{1}{2} g l_{r}-\frac{1}{2} \dot{v}_{x} h+\frac{l_{r}}{b_{f}} \dot{v}_{y} h\right) \\
& F_{z r l}=\frac{m}{l_{f}+l_{r}}\left(\frac{1}{2} g l_{f}+\frac{1}{2} \dot{v}_{x} h-\frac{l_{f}}{b_{r}} \dot{v}_{y} h\right) \\
& F_{z r l}=\frac{m}{l_{f}+l_{r}}\left(\frac{1}{2} g l_{f}+\frac{1}{2} \dot{v}_{x} h+\frac{l_{f}}{b_{r}} \dot{v}_{y} h\right)
\end{aligned}
$$

where $h$ is the height of the vehicle CG above the ground. It should be noted that the load transfer effect is important during the overtaking scenario because the high velocity may lead to the serious load transfer effect when turning. This may compromise the vehicle dynamics stability.

The non-linear Dugoff tyre model is used in this paper [22], and is described by:

$$
\begin{gathered}
\lambda_{i}=\frac{\mu F_{z i}\left[1-\varepsilon_{r} u_{i} \sqrt{s_{i}^{2}+\tan ^{2} \alpha_{i}}\right]\left(1-s_{i}\right)}{2 \sqrt{C_{s}^{2} s_{i}^{2}+C_{\alpha}^{2} \tan ^{2} \alpha_{i}}} \\
f\left(\lambda_{i}\right)= \begin{cases}\lambda_{i}\left(2-\lambda_{i}\right) & \left(\lambda_{i}<1\right) \\
1 & \left(\lambda_{i}>1\right)\end{cases} \\
F_{s i}=\frac{C_{\alpha} \tan \alpha_{i}}{1-s_{i}} f\left(\lambda_{i}\right) \\
F_{t i}=\frac{C_{s} s_{i}}{1-s_{i}} f\left(\lambda_{i}\right)
\end{gathered}
$$

$u_{i}$ is the vehicle velocity component in the wheel plane which is defined for each wheel as:

$$
\begin{aligned}
& u_{f l}=\left(v_{x}+\frac{1}{2} b_{f} r\right) \cos \delta_{f l}+\left(v_{y}+l_{f} r\right) \sin \delta_{f l} \\
& u_{f r}=\left(v_{x}-\frac{1}{2} b_{f} r\right) \cos \delta_{f r}+\left(v_{y}+l_{f} r\right) \sin \delta_{f r} \\
& u_{r l}=\left(v_{x}+\frac{1}{2} b_{r} r\right) \cos \delta_{r l}-\left(l_{r} r-v_{y}\right) \sin \delta_{r l}
\end{aligned}
$$




$$
u_{r l}=\left(v_{x}-\frac{1}{2} b_{r} r\right) \cos \delta_{r r}-\left(l_{r} r-v_{y}\right) \sin \delta_{r r}
$$

The wheel rotation dynamics is described by the following equation:

$$
I_{\omega} \dot{\omega}_{i}=-R_{\omega} F_{t i}+T_{i}
$$

\section{Potential FiELD MethoD}

In the autonomous vehicle steering control, the potential field includes the component that guides the vehicle towards the desired path and the obstacle potentials induced by the road curb or other vehicles in the traffic. The total potential energy function can be presented by the following equation:

$$
U=U_{\text {att }}+U_{\text {rep }}+U_{s}
$$

where $U_{a t t}$ is the attractive potential that guides the vehicle towards the desired path and $U_{r e p}$ is the repulsive potential that guides the vehicle away from the obstacle. $U_{S}$ is the potential function that minimise the yaw angle change rate, which can improve the vehicle handling and stability performance:

$$
U_{s}=c(\varphi(k+1)-\varphi(k))^{2}
$$

where $\varphi(k)$ and $\varphi(k+1)$ present the yaw angle in the $k t h$ time step and $(k+1) t h$ time step. $c$ is the scaling factor.

The attractive potential $U_{a t t}$ can be presented by (9) according to [11]:

$$
U_{\text {att }}=\alpha_{p}\left\|p_{\text {tar }}(k)-p(k)\right\|^{r}+\alpha_{v}\left\|v_{t a r}(k)-v(k)\right\|^{n}
$$

where $p(k)$ and $p_{\operatorname{tar}}(k)$ denote the position of the vehicle and the desired path at time $t . v_{t a r}(k)$ and $v(k)$ present the actual velocity of the vehicle and the desired velocity of the trajectory at time step $k$, which includes the longitudinal velocity and lateral velocity. $\alpha_{p}$ and $\alpha_{v}$ are scalar positive parameters. $r$ and $n$ are positive parameters. In the actual vehicle dynamics control, the vehicle velocity can be easily controlled by achieving the desired longitudinal velocity and yaw angle. However, the vehicle position error is hard to be controlled for the on-road vehicle. The control of the lateral and longitudinal position tracking error requires the control of the longitudinal and lateral forces. For the in-door robot such as the holonomic omni-directional robot, the orientation and position can be controlled independently and consequently the longitudinal position and lateral position can be perfectly tracked without interfering with the yaw angle. However, for the autonomous electric vehicle, the longitudinal force and lateral force will have strong couple effect on the yaw angle of the vehicle during the position tracking due to the limitation of the vehicle mobility. Therefore, in this study, the desired trajectory is only tracked by the desired vehicle velocity and (9) can be rewritten as follows by assuming $n=2$ :

$$
\begin{gathered}
U_{a t t}=\alpha_{v}\left\|v_{t a r}(k)-v(k)\right\|^{2}=\alpha_{v}\left(v_{x}(k) \tan \varphi(k)-\right. \\
\left.v_{y d-g}(k)\right)^{2}
\end{gathered}
$$

where $v_{x}$ is the longitudinal velocity in the vehicle body-fixed coordinate system, and this value is transferred into the global coordinate system as the lateral velocity by multiplying $\tan \varphi$. $v_{y d-g}$ is the desired vehicle lateral velocity of the central line of the desired trajectory in the global coordinate system, and this value is obtained according to the derivative of the lateral position of the central line of the trajectory. The actual vehicle longitudinal velocity is assumed to track the desired value accurately due to the application of the trajectory controller in Section 4, so it is not included in (10).

In addition, to determine the repulsive potential function of the road, the road boundary trajectory should be determined at first. The on-board sensors and camera can obtain the information of the road boundary ahead of the vehicle [23]. In addition, the real-time vehicle states (such as longitudinal velocity, lateral velocity and yaw rate) are assumed to be known or measurable since a number of studies have proposed various vehicle state estimation methods [24][25][26]. The road boundary can be described by the line of the upper boundary and the line of the lower boundary:

$$
\begin{aligned}
& Y_{u b}=f_{1}(X) \\
& Y_{l b}=f_{2}(X)
\end{aligned}
$$

where $X$ is the longitudinal position and $Y_{u b}\left(Y_{l b}\right)$ is the lateral position of the boundary in the global coordinate system.

This road boundary position function depends on the position. In order to guarantee the vehicle is moving within the road boundary, the repulsive potential function is determined by the distance between the current vehicle lateral position and lateral positions of the corresponding upper and lower boundary when their longitudinal positions are same. However, the vehicle motion equations in Section II are usually described by the function depending on time in the vehicle body-fixed coordinate system. Thus, the following equations are used to transfer the time-dependent vehicle motion equation in the body-fixed coordinate system into the position-dependent vehicle motion equation in the global coordinate system:

$$
\begin{array}{r}
X(k+1)=X(k)+\Delta t\left(v_{x}(k) \cos \varphi(k)-v_{y}(k) \sin \varphi(k)\right) \\
Y(k+1)=Y(k)+\Delta t\left(v_{x}(k) \sin \varphi(k)+v_{y}(k) \cos \varphi(k)\right)
\end{array}
$$

where $k$ presents the number of time step. $\Delta t$ is the length of each time step and can be presented by the difference between the time value of the next time step $t(k+1)$ and the current time step $t(k)$ :

$$
\Delta t=t(k+1)-t(k)
$$

$v_{x}$ is the longitudinal velocity in the vehicle body-fixed coordinate system and $v_{y}$ is the lateral velocity in the vehicle body-fixed coordinate system.

The boundary condition of the vehicle motion can be presented by the following equation:

$$
Y_{u b}(X(k+1)) \leq Y(k+1) \leq Y_{l b}(X(k+1))
$$

Thus, the repulsive potential function can be determined according to the boundary condition (14). When the vehicle lateral position is between the central line and upper boundary, the repulsive potential function is as follows:

$$
U_{\text {rep }}=\frac{b_{1}}{\left(Y(k+1)-Y_{u b}(X(k+1))\right)^{2}}
$$


When the vehicle lateral position is between the central line and the lower boundary, the repulsive potential function is as follows:

$$
U_{\text {rep }}=\frac{b_{2}}{\left(Y(k+1)-Y_{l b}(X(k+1))\right)^{2}}
$$

where $b_{1}, b_{2}$ are the scaling factors.

In addition to the road boundary, vehicle velocity constraints should be considered. Assume the velocity of the controlled vehicle is constrained by $v_{\min }$ and $v_{\max }$, respectively. The value of $v_{\min }$ is usually zero unless there is a low speed limit in the highway. The value of $v_{\max }$ is usually constrained by the high speed limit in the highway.

The optimal control of vehicle motion can be achieved by choosing the optimal value of desired yaw angle to minimise the total potential energy function $U$. Thus, the cost function of the optimisation problem can be presented as follows:

$$
\begin{aligned}
J_{1 \text { min }, \varphi_{d}, v_{x}}=a_{0}( & \left.v_{x d}-v_{x}\right)^{2} \\
& +\alpha_{v}\left(v_{x} \tan \varphi_{d}(k+1)-v_{y d-g}(k+1)\right)^{2} \\
& +\frac{b_{1}}{\left(Y(k+1)-Y_{u b}(X(k+1))\right)^{2}} \\
& +\frac{b_{2}}{\left(Y(k+1)-Y_{l b}(X(k+1))\right)^{2}} \\
& +c\left(\varphi_{d}(k+1)-\varphi_{d}(k)\right)^{2}
\end{aligned}
$$

s.t.

$$
\begin{aligned}
-\frac{\pi}{2} & \leq \varphi_{d} \leq \frac{\pi}{2} \\
v_{\min } & \leq v_{x} \leq v_{\max }
\end{aligned}
$$

where $X(k+1)$ and $Y(k+1)$ can be determined by (12). $a_{0}$ is the scaling factor related to the term of achieving the desired longitudinal velocity. This optimisation problem can be solved by various algorithms. In this paper, the Matlab embedded function ' $\mathrm{fmincon}$ ' is applied to solve this problem and obtain the desired yaw angle $\varphi_{d}$. In the next section, the vehicle dynamics trajectory controller is proposed to track this desired yaw angle.

\section{VEHICLE TRAJECTORY CONTROLLER}

Based on the desired vehicle longitudinal velocity and desired yaw angle of the trajectory, the autonomous vehicle motion can be controlled. The vehicle tracking error dynamics equation can be presented by the following equation based on [12]:

$$
\begin{gathered}
\dot{\tilde{e}}_{y}=v_{x} \sin \left(\tilde{\varphi}+\varphi_{d}\right)+v_{y} \cos \left(\tilde{\varphi}+\varphi_{d}\right) \\
\tilde{v}_{x}=\left[v_{x} \cos \left(\tilde{\varphi}+\varphi_{d}\right)-v_{y} \sin \left(\tilde{\varphi}+\varphi_{d}\right)\right]-v_{x d} \\
\tilde{\varphi}=\varphi_{a c t}-\varphi_{d}
\end{gathered}
$$

where $\varphi_{a c t}$ and $\varphi_{d}$ are the vehicle's actual and desired yaw angles, respectively. $\dot{\tilde{e}}_{y}$ is the derivative of the lateral position error in the global coordinate system. $\tilde{v}_{x}$ is the error between the vehicle actual longitudinal velocity and the desired value $v_{x d}$ tangential to the path in the global coordinate system.

The vehicle trajectory controller includes two parts of: the feedforward controller and the feedback controller. The feedforward force and moment demands are calculated with the assumption that vehicle follows the desired trajectory:

$$
\begin{gathered}
F_{t, \text { feedforward }}=m \dot{v}_{x d} \\
F_{n, \text { feedforward }}=m v_{x d} \dot{\varphi}_{d} \\
M_{z, \text { feedforward }}=I_{z} \ddot{\varphi}_{d}
\end{gathered}
$$

where $F_{t, \text { feedforward }}$ is the total force demand in the global coordinate frame tangential to the path in the feedforward controller. $F_{n, \text { feedforward }}$ is the total force demand in the global coordinate frame norm to the path in the feedforward controller. $M_{z, \text { feedforward }}$ is the total yaw moment required to achieve the desired vehicle motion in the feedforward controller. The feedforward controller (18) requires the perfectly tracking of the desired trajectory, which is unrealistic in the actual vehicle control.

To compensate the tracking error in the feedforward control, the feedback controller is proposed. The feedback force and moment demands are calculated by the following equations:

$$
\begin{gathered}
F_{t, \text { feedback }}=-m \dot{\tilde{e}}_{y} \dot{\varphi}_{d}-K_{1} \tilde{v}_{x} \\
F_{n, \text { feedback }}=m \tilde{v}_{x} \dot{\varphi}_{d}-K_{2 d} \dot{\tilde{e}}_{y}-K_{2 p} \tilde{e}_{y} \\
M_{z, \text { feedback }}=-K_{3 d} \dot{\tilde{\varphi}}-K_{3 p} \tilde{\varphi}
\end{gathered}
$$

where $K_{1}, K_{2 d}, K_{2 p}, K_{3 d}, K_{3 p}$ are feedback control gains. $F_{t, \text { feedback }}$ is the total force demand in the global coordinate frame tangential to the path in the feedback controller. $F_{n, \text { feedback }}$ is the total force demand in the global coordinate frame norm to the path in the feedback controller. $M_{z, \text { feedback }}$ is the total yaw moment required to achieve the desired vehicle motion in the feedback controller.

When the vehicle is perfectly tracking the desired path, the total feedforward and feedback force tangential to the path $F_{t, \text { total }}=F_{t, \text { feedforward }}+F_{t, \text { feedback }}$ should be equal to the total longitudinal force of the vehicle $F_{x, \text { total }}$ and the total feedforward and feedback force norm to the path $F_{n, t o t a l}=$ $F_{n, \text { feedforward }}+F_{n \text {,feedback }}$ should be equal to the total lateral force of the vehicle $F_{y, \text { total }}$. When the tracking error is considered, however, the total demand forces in the global coordinate frame should be transferred into the vehicle body-fixed coordinate frame by the following equations:

$$
\begin{gathered}
F_{x, \text { total }}=F_{t, \text { total }} \cos \tilde{\varphi}+F_{n, \text { total }} \sin \tilde{\varphi} \\
F_{y, \text { total }}=-F_{t, \text { total }} \sin \tilde{\varphi}+F_{n, \text { total }} \cos \tilde{\varphi} \\
M_{z, \text { total }}=M_{z, \text { feedford }}+M_{z, \text { feedback }}
\end{gathered}
$$

In this section, in order to achieve the trajectory tracking control, the vehicle position error is described in the global coordinate frame at first. After that, according to the position error, the demand total longitudinal force and lateral force should be transferred from the global coordinate frame into the vehicle body-fixed coordinate frame. This is because that the vehicle dynamics control can only be achieved in the body-fixed coordinate system. In the following section, the 
steering and driving actuators are controlled to achieve the desired total longitudinal force, the total lateral force and yaw moment.

\section{Optimal Actuator CONTROl Allocation Method}

In this study, the 4WIS-4WID electric vehicle is used to achieve the desired trajectory control. This 4WIS-4WID electric vehicle has the advantage of using redundant control actuators to achieve better control performance.

In this section, the control targets of the actuator control allocation method are the desired total longitudinal tyre force, the desired total lateral tyre force and desired yaw moment determined in the upper level trajectory controller in the last section. In addition, the individual allocated tyre forces are minimised to guarantee each tyre has been used sufficiently. The cost function of this actuator control allocation problem is shown as follows:

$$
\begin{gathered}
J_{2 m i n, F_{t i}, F_{s i}}=\frac{F_{t f l}^{2}+F_{s f l}^{2}}{\mu^{2} F_{z f l}^{2}}+\frac{F_{t f r}^{2}+F_{s f r}^{2}}{\mu^{2} F_{z f r}^{2}}+\frac{F_{t r l}^{2}+F_{s r l}^{2}}{\mu^{2} F_{z r l}^{2}} \\
+\frac{F_{t r r}^{2}+F_{s r r}^{2}}{\mu^{2} F_{z r r}^{2}}
\end{gathered}
$$

subject to:

$$
\begin{gathered}
F_{t f l} \cos \delta_{f l}+F_{t f r} \cos \delta_{f r}+F_{t r l} \cos \delta_{r l}+F_{t r r} \cos \delta_{r r}-F_{s f l} \sin \delta_{f l}- \\
F_{s f r} \sin \delta_{f r}-F_{s r l} \sin \delta_{r l}-F_{s r r} \sin \delta_{r r}=F_{x, t o t a l}-K_{s 1} \operatorname{Sat}\left(F_{x}-F_{x, \text { total }}\right) \\
F_{t f l} \sin \delta_{f l}+F_{t f r} \sin \delta_{f r}+F_{t r l} \sin \delta_{r l}+F_{t r r} \sin \delta_{r r}+F_{s f l} \cos \delta_{f l}+ \\
F_{s f r} \cos \delta_{f r}+F_{s r l} \cos \delta_{r l}+F_{s r r} \cos \delta_{r r}=F_{y, t o t a l}-K_{s 2} \operatorname{Sat}\left(F_{y}-F_{y, t o t a l}\right) \\
F_{t f l}\left(l_{f} \sin \delta_{f l}+0.5 b_{f} \cos \delta_{f l}\right)+F_{t f r}\left(l_{f} \sin \delta_{f r}-0.5 b_{f} \cos \delta_{f r}\right) \\
+F_{t r l}\left(-l_{r} \sin \delta_{r l}+0.5 b_{r} \cos \delta_{r l}\right) \\
+F_{t r r}\left(-l_{r} \sin \delta_{r r}-0.5 b_{r} \cos \delta_{r r}\right) \\
+F_{s f l}\left(l_{f} \cos \delta_{f l}-0.5 b_{f} \sin \delta_{f l}\right) \\
+F_{s f r}\left(l_{f} \cos \delta_{f r}+0.5 b_{f} \sin \delta_{f r}\right) \\
+F_{s r l}\left(-l_{r} \cos \delta_{r l}-0.5 b_{r} \sin \delta_{r l}\right) \\
+F_{s r r}\left(-l_{r} \cos \delta_{r r}+0.5 b_{r} \sin \delta_{r r}\right) \\
\\
=M_{z, t o t a l}-K_{s 3} \operatorname{Sat}\left(M_{z}-M_{z, t o t a l}\right)
\end{gathered}
$$

where $F_{x}, F_{y}$ are the actual total longitudinal tyre force and lateral tyre force. $M_{z}=I_{z} \dot{r}$ is the actual yaw moment of the vehicle.

$$
\begin{gathered}
F_{t i}^{2}+F_{s i}^{2} \leq \mu F_{z i}^{2} \\
-\frac{T_{b \max }}{R_{\omega}} \leq F_{t i} \leq \frac{T_{d \max }}{R_{\omega}}
\end{gathered}
$$

The constraints (21a), (21b) and (21c) are applied here to achieve the desired longitudinal tyre force, lateral tyre force and yaw moment. To overcome the distribution error due to the non-linear characteristic of the vehicle dynamics model, the sliding mode controller (SMC) is proposed in constraints (21a), (21b) and (21c) to accurately tracking the desired values. The effect of tyre friction circle is considered in (21d) and the constraint of the individual wheel driving/braking actuator is shown in (21e). In this study, an in-wheel brushless DC electric motor is applied. It has been suggested that the maximum driving torque $T_{d m a x}$ is $100 \mathrm{~N} . \mathrm{m}$ and the maximum regenerated brake torque $T_{\text {bmax }}$ is $80 \mathrm{~N} . \mathrm{m}$ [27]. The optimisation problem (21) can also be solved by the Matlab embedded function 'fmincon' and the detailed analysis of the optimisation algorithm is beyond the scope of this study.

When the individual tyre forces have been allocated in (21), the controlled value of individual actuator can be mapped from the individual tyre force by the following equations:

$$
\begin{gathered}
T_{i}=F_{t i} R_{\omega} \\
\delta_{f l}=\frac{F_{s f l}}{C_{\alpha}}+\frac{l_{f} r}{v_{x}} \\
\delta_{f r}=\frac{F_{s f r}}{C_{\alpha}}+\frac{l_{f} r}{v_{x}} \\
\delta_{r l}=\frac{F_{s r l}}{C_{\alpha}}-\frac{l_{r} r}{v_{x}} \\
\delta_{r r}=\frac{F_{s r r}}{C_{\alpha}}-\frac{l_{r} r}{v_{x}}
\end{gathered}
$$

The practical limitation of the steering angle is considered between -90 degrees and 90 degrees $\left(\delta_{\max }=90\right)$, which is larger than the traditional vehicle [28]. Thus,

$$
-\delta_{\max } \leq \delta_{i} \leq \delta_{\max }
$$

The whole control structure of the proposed potential field based trajectory tracking controller is shown in Figure 1.

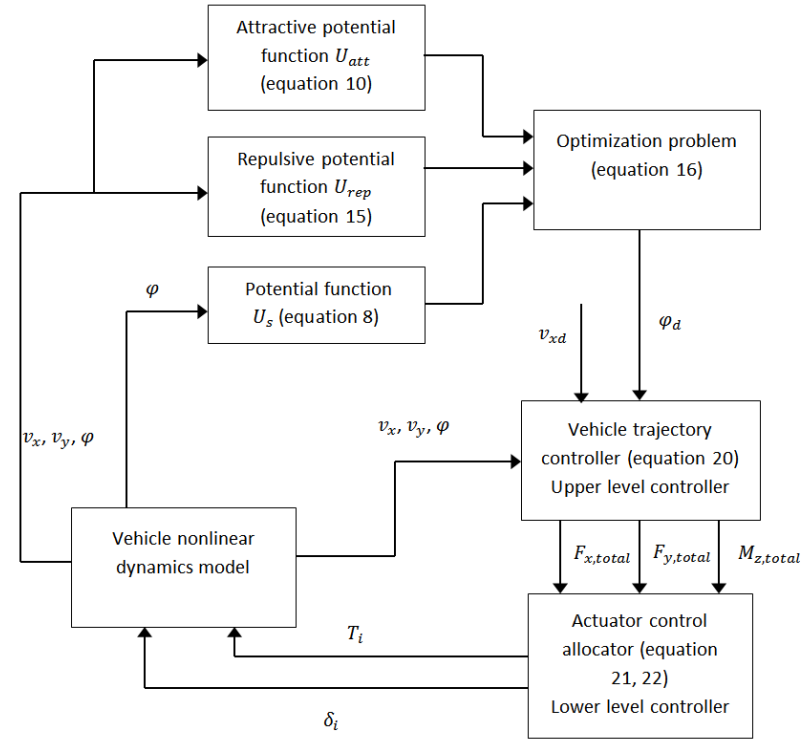

Fig. 1. The whole control structure of the proposed potential field based trajectory controller.

\section{Simulation ReSUlts}

In this section, four sets of simulations are used to test the effectiveness of the proposed vehicle trajectory controller. In the first set of simulations, the road boundary is wide enough and the boundary constraints can be neglected. The control targets are the tracking of the road central line and minimising of the yaw rate to guarantee the smoothness of the trajectory. In the second set of simulations, the road boundary is much narrow than the first set of simulations and the boundary avoiding control is the primary control target. In the third and fourth set of simulations, the desired vehicle path and road boundary are changing with the actual traffic condition. The simulation parameters are shown in Table 1.

TABLE I

PARAMETER VALUES USED IN SIMULATIONS. [20] 


\begin{tabular}{cll}
\hline \hline Symbol & \multicolumn{1}{c}{ Definition } & \multicolumn{1}{c}{ Values } \\
\hline$m$ & $\begin{array}{l}\text { Vehicle mass } \\
\text { Distance of c.g. from } \\
l_{f}\end{array}$ & $\begin{array}{l}1298.9 \mathrm{mg} \\
\text { the front axle }\end{array}$ \\
$l_{r}$ & $\begin{array}{l}\text { Distance of c.g. from } \\
\text { the rear axle }\end{array}$ & $1.454 \mathrm{~m}$ \\
$b_{f}$ & $\begin{array}{l}\text { Front track width } \\
b_{r}\end{array}$ & $1.436 \mathrm{~m}$ \\
$C_{s}$ & $\begin{array}{l}\text { Rear track width } \\
\text { Longitudinal stiffness }\end{array}$ & $1.436 \mathrm{~m}$ \\
$I_{z}$ & $\begin{array}{l}\text { Vehicle moment of } \\
\text { inertial about yaw axle }\end{array}$ & $1627 \mathrm{kgm}{ }^{2}$ \\
$R_{\omega}$ & $\begin{array}{l}\text { Wheel radius } \\
I_{\omega}\end{array}$ & $\begin{array}{l}\text { Wheel moment of } \\
\text { inertial }\end{array}$ \\
$\varepsilon_{r}$ & $\begin{array}{l}\text { Road adhesion } \\
\text { reduction factor } \\
\text { Cornering stiffness of } \\
C_{\alpha}\end{array}$ & $2.1 \mathrm{kgm} \mathrm{m}^{2}$ \\
& the tyre & $0.015 \mathrm{~s} / \mathrm{m}$ \\
\hline \hline
\end{tabular}

In the first set of simulations, the upper level boundary, road centre line and lower level boundary of vehicle desired trajectory are presented in Figure 3. In this simulation, the vehicle initial velocity is $20 \mathrm{~m} / \mathrm{s}$ and the tyre-road friction coefficient is 0.9. It should be noted that the upper and lower boundaries in the simulation indicate the constraints of the vehicle C.G. point and the vehicle geometric length is neglected here.

Figure 2 presents the simulation results when the road centre line is strictly followed. This means $c=b_{1}=b_{2}=0, a_{v}=$ $100, a_{0}=0$ in the optimal control law (16). In Figure 3, the vehicle trajectory is optimised by the minimising of the position error and the minimising of the desired yaw rate. This means that $b_{1}=b_{2}=0$ and $a_{0}=0, a_{v}=1, c=80000$ in the optimal control law (16). In Figure 3, the road centre line is roughly followed by the actual vehicle and the vehicle trajectory is much smoother than the trajectory in Figure 2. Figure 4 and Figure 5 compare vehicle longitudinal velocity, body slip angle responses and yaw rate responses when the desired trajectory is strictly followed (Figure 2) and when the vehicle trajectory is optimised (Figure 3). It can be found that if the centre line of the path is strictly followed, the actual longitudinal velocity is varying relatively bigger, and the vehicle body slip and yaw rate oscillate significantly. The vehicle handling and stability performance would be significantly impaired and the vehicle is moving in a dangerous condition. When the actual trajectory is optimised, vehicle body slip angle is much smaller and the vehicle yaw rate and longitudinal velocity response are more stable. This proves that the proposed potential field method can successfully improve the vehicle handling and stability performance, which are generally defined in terms of vehicle yaw rate and body slip angle.

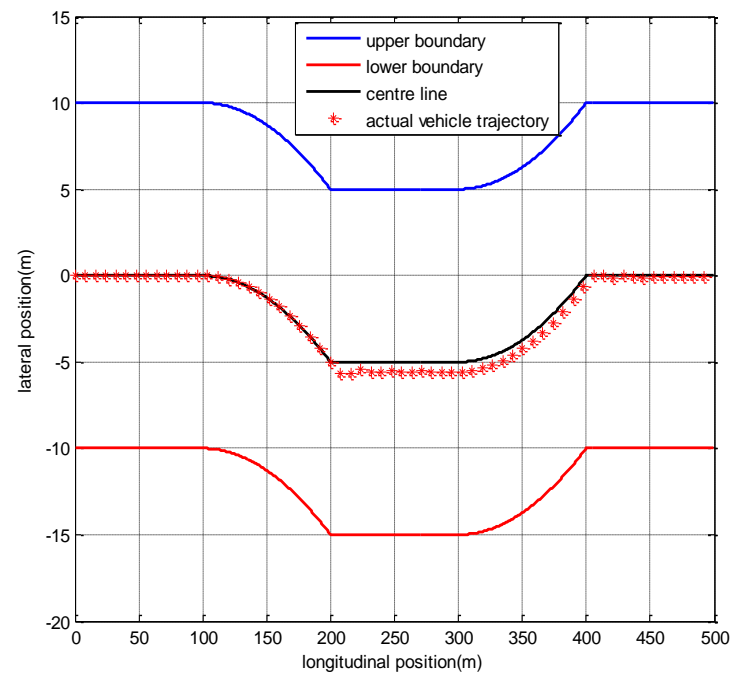

Fig. 2. The actual vehicle trajectory when the desired trajectory is strictly followed in the first set of simulations.

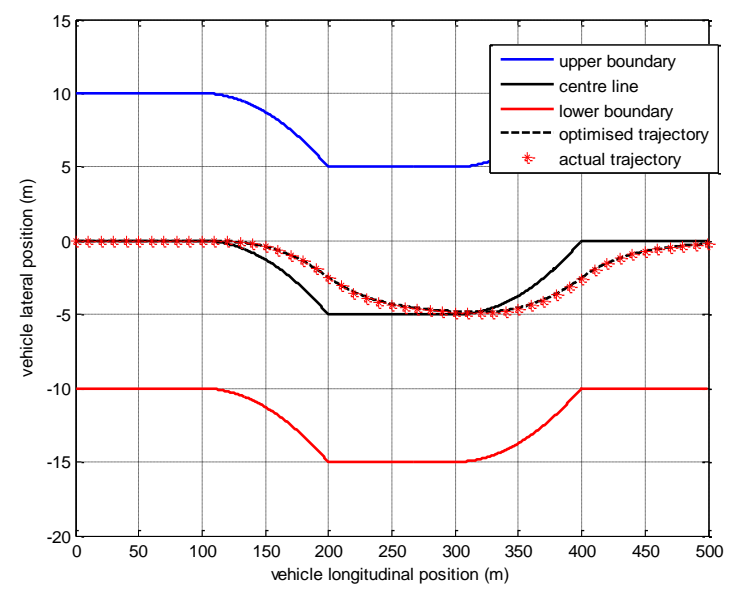

Figure 3. The actual vehicle trajectory when the trajectory of the path is optimised in the first set of simulations.

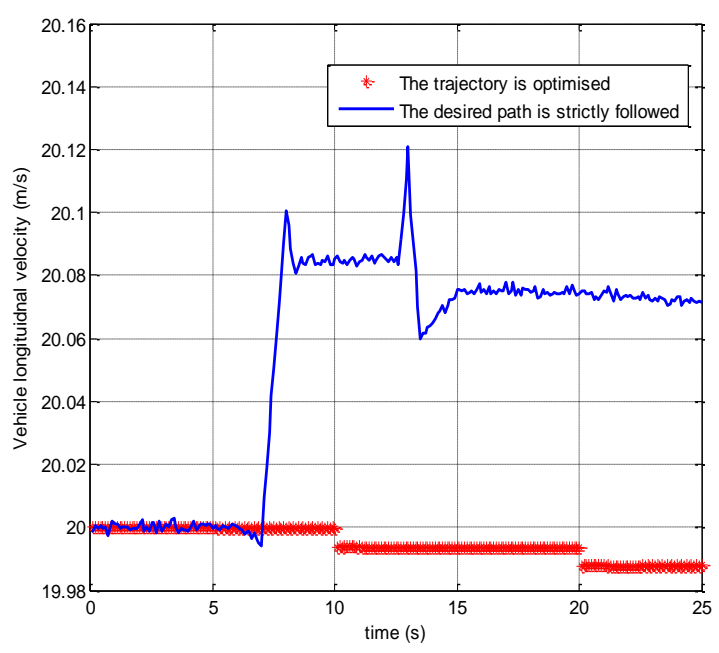

Figure 4. The actual vehicle velocity in the first set of simulations. 


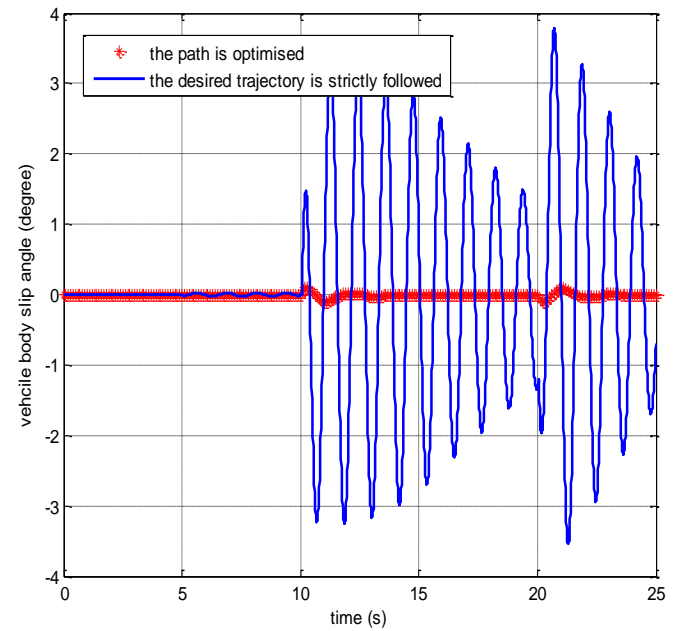

(a)

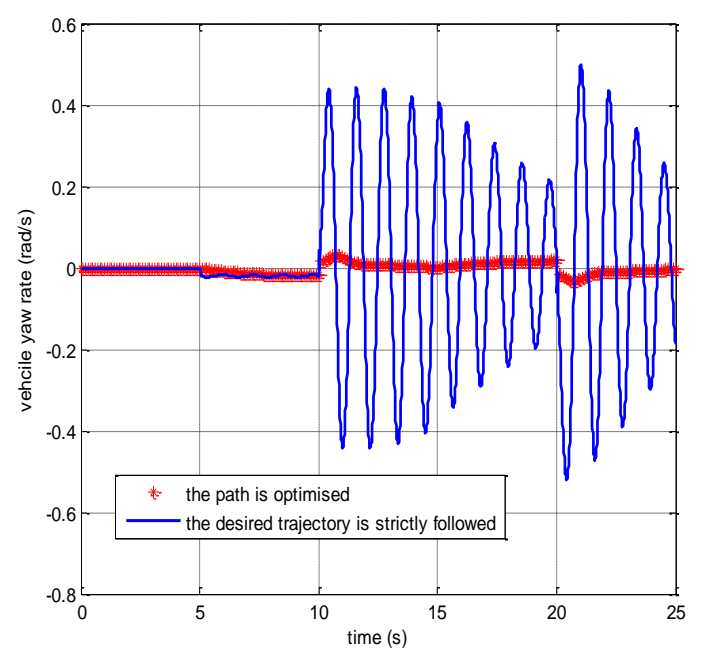

(b)

Figure 5. The actual vehicle responses (a) body slip angle (b) yaw rate in the first set of simulations.

In the second set of simulations, the vehicle initial velocity and tyre-road friction coefficient remain unchanged. According to Figure 6, the desired trajectory and road boundary are more challenge than the first set of simulation. This path simulates the situation when the vehicle is trying to avoid the obstacle by doing the double lane change.

The results in Figure 6 demonstrate the potential field method can successfully avoid the road boundary in the narrow moving space by minimising the yaw rate and not strictly following the road centre line. In this case, $a_{0}=0, a_{v}=0.5, b_{1}=b_{2}=$ $2000, c=20000$ are used in the optimal law (16). This is quite different from the simple trajectory-following method as shown in Figure 7. It can be found in Figure 7 that the vehicle hits the lower boundary after the first turning and this is a serious problem that the vehicle may have an accident. It is noted that in the actual implementation of trajectory controller and actuator controller, there are a number of reasons that may cause the control error, such as the highly nonlinear vehicle dynamics model (which is used to represent a more realistic vehicle in practice) used in the simulation, the constraints of control actuators and the selection of controller gains. This control error causes the difference between the actual trajectory and the optimised one in (16), which can be observed in Figure 6.

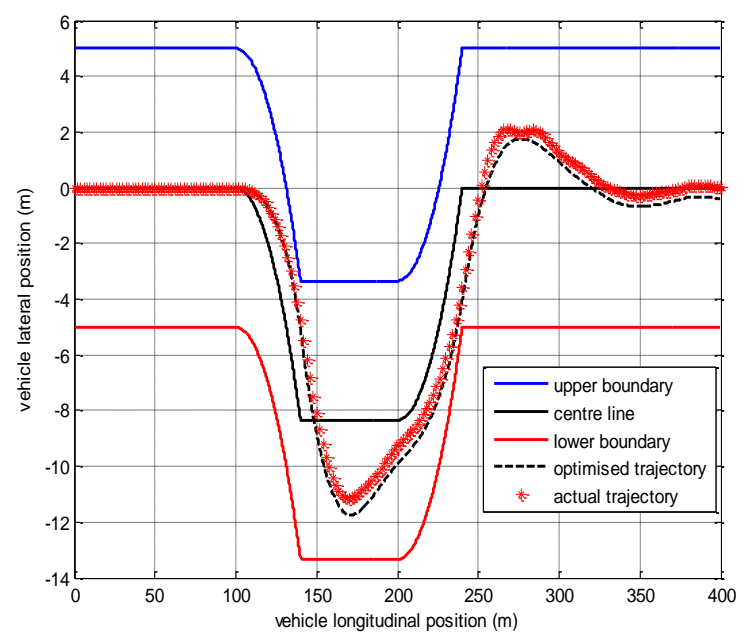

Fig. 6. The actual vehicle trajectory when the trajectory of the path is optimised in the second set of simulations.

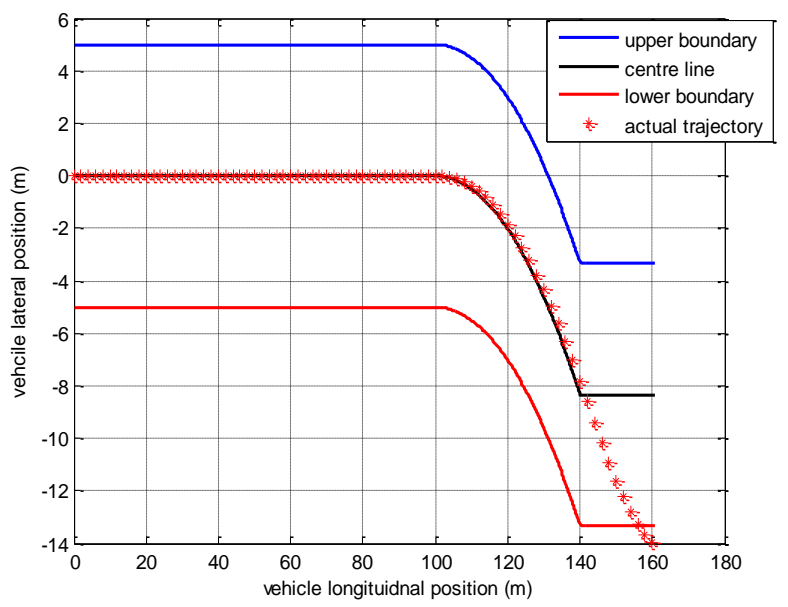

Fig. 7. The actual vehicle trajectory when the desired trajectory is strictly followed in the second set of simulations.

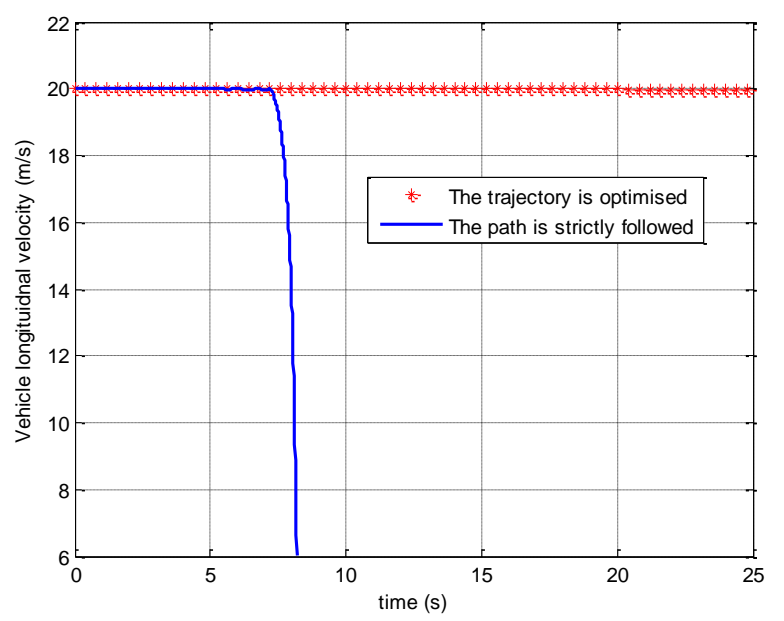

Fig. 8. The actual vehicle longitudinal velocity in the second set of simulations. 


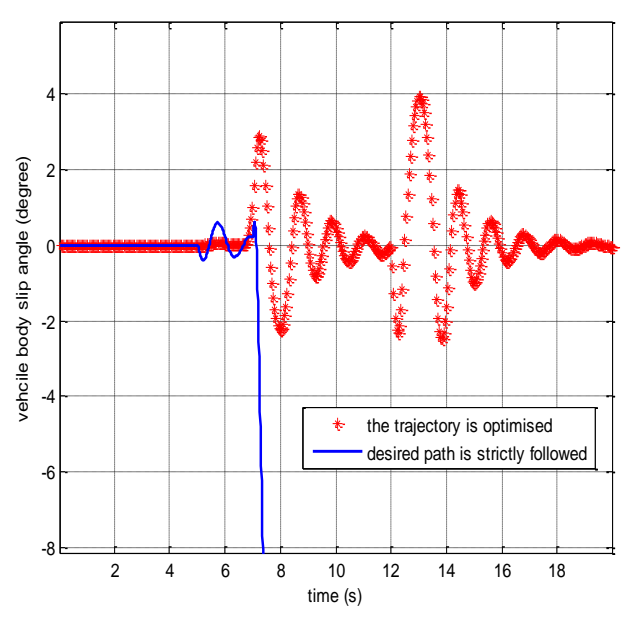

(a)

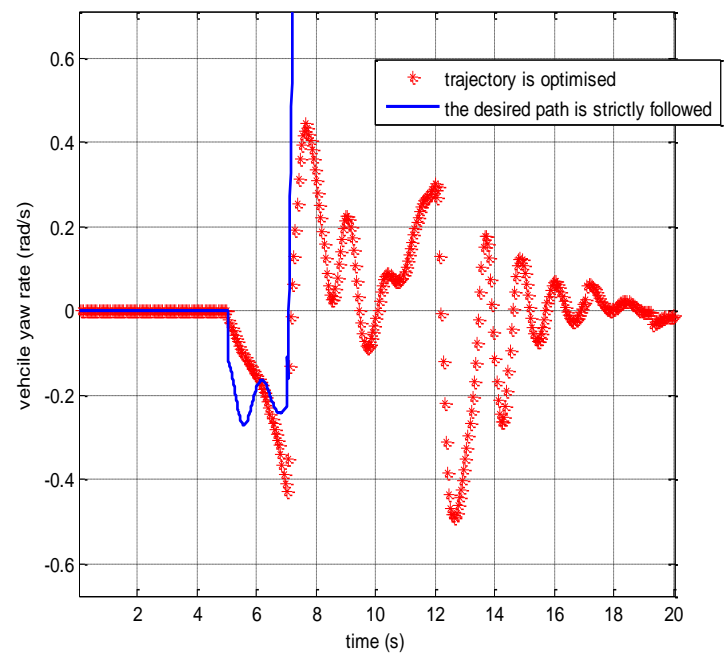

(b)

Figure 9. The actual vehicle responses (a) body slip angle (b) yaw rate in the second set of simulations.

Figure 8 and Figure 9 compare the actual vehicle longitudinal velocity, body slip angle responses and yaw rate responses when the desired path is strictly followed and when the trajectory is optimised. When the desired path is strictly followed, the longitudinal velocity decreases rapidly and the body slip angle and the yaw rate are highly unstable when the vehicle hits the lower boundary. The main reason for the instability of the vehicle in Figure 7 is that the only control target for the strict path-following method is that the desired yaw angle and longitudinal velocity must strictly follow the desired path. When the vehicle is turning in a narrow angle, the large change rate of the yaw angle is required if the desired path is strictly followed. This large change rate of yaw angle as shown in Figure 9(b) results in the instability of the vehicle. When the proposed potential field method is applied to optimise the vehicle trajectory, the vehicle body slip angle and yaw rate performance are much improved.

Table 2 summaries the maximum vehicle longitudinal velocity when the vehicle is turning with certain turning radius without hitting the road boundary and this value is obtained by a number of simulation tests. According to Table 2, the vehicle maximum velocity increases when the turning radius increases. In addition, the boundary optimisation gains $\left(b_{1}, b_{2}\right)$ also increase and play an important role when the turning radius is small.

TABLE II VEHICLE MAXIMUM LONGITUDINAL VELOCITY DURING VEHICLE TURNING

\begin{tabular}{|c|c|c|}
\hline $\begin{array}{l}\text { Turn radius } \\
(\mathrm{m})\end{array}$ & $\begin{array}{l}\text { Maximum longitudinal } \\
\text { velocity }(\mathrm{m} / \mathrm{s})\end{array}$ & Optimisation control gains \\
\hline 1000 & $>50$ & $\begin{array}{l}b_{1}=b_{2}=0 \text { and } a_{v}=1, c= \\
80000, a_{0}=0\end{array}$ \\
\hline 200 & 25 & $\begin{array}{l}a_{v}=0.5, b_{1}=b_{2}=0, c \\
=20000, a_{0}=0\end{array}$ \\
\hline 100 & 20 & $\begin{array}{l}a_{v}=0.5, b_{1}=b_{2}=2000, c \\
=20000, a_{0}=0\end{array}$ \\
\hline
\end{tabular}

It should be noted that the scaling factor $a_{0}$ in the optimisation problem (16) is assumed as zero in the first two sets of simulations because the longitudinal velocity is not required to achieve the certain value and the controller tries to maintain the initial velocity. In addition, the velocity constraint (16b) is also neglected here since there are no front and rear vehicles.

In the third set of the simulations, the impacts of the surrounding traffic of the controlled autonomous vehicle are considered. The vehicle initial velocity and tyre-road friction coefficient remain unchanged. The vehicle is assumed to move along the desired path with wide boundary as Figure 2 at the beginning. After 200 meters in the longitudinal direction, the road boundary is narrower than the boundary at the beginning due to the effect of the surrounding traffic. In this simulation, $a_{0}=0, a_{v}=0.5, b_{1}=b_{2}=0, c=20000$ are used in the optimal law (16). In addition, it is assumed that there exists a vehicle in front of the controlled vehicle and a vehicle behind the controlled vehicle along the path, which is quite common in the real situation. The controlled vehicle longitudinal positon in the body-fixed coordinate system is constrained by the positions of the front vehicle and rear vehicle on the road to avoid collision, which is expressed as the following additional road boundary conditions:

$$
\begin{aligned}
& \left|X_{1}-X_{2}\right|>d \\
& \left|X_{1}-X_{3}\right|>d
\end{aligned}
$$

where $X_{1}$ is the longitudinal position of the controlled vehicle. $X_{2}$ and $X_{3}$ are the longitudinal positions of the front vehicle and rear vehicle, respectively. The longitudinal positions of the front and rear vehicles $X_{2}, X_{3}$ can be calculated as:

$$
\begin{aligned}
& X_{2}=X_{20}+v_{2} t \\
& X_{3}=X_{30}+v_{3} t
\end{aligned}
$$

where $X_{20}$ and $X_{30}$ are the initial longitudinal positions of the front and rear vehicles, respectively. $v_{2}$ and $v_{3}$ are the longitudinal velocities of the front and rear vehicles, which are assumed as the constant values. $d$ is a certain safety distance. Generally, a larger safety distance indicates that the controlled autonomous vehicle can have wider moving corridor. With a wider moving corridor, a more smooth vehicle trajectory can be optimised by the proposed potential field method and 
consequently the stability of the controller can be further improved.

Figure 10 suggests that the vehicle is controlled within the road boundary. Figure 11 shows that the longitudinal position of the controlled vehicle is between the longitudinal positions of front vehicle and rear vehicle with certain distance along the road. The above simulation results prove that the controlled vehicle can satisfy all the boundary constraints and velocity constraints and the desired trajectory is successfully achieved. Figure 12 suggests that the vehicle yaw rate and body slip angle change abruptly during the turning.

In the fourth set of simulations, the proposed potential field controller is applied in the actual traffic condition of overtaking and lane change. The tyre-road friction coefficient is assumed to be unchanged. At the beginning, the controlled vehicle is assumed to move on the bottom lane of the highway with the longitudinal velocity of $18 \mathrm{~m} / \mathrm{s}$, while another vehicle is moving on the top lane of the highway with velocity of $20 \mathrm{~m} / \mathrm{s}$. In order to overtake the vehicle in the top lane at 5 seconds, the controlled vehicle should turn on the left-turning signal, and then start to increase the speed into $20 \mathrm{~m} / \mathrm{s}$ and make the lane change. At the same time of 5 seconds, when the driver of the top lane vehicle notices the left-turning signal of overtaking vehicle, he may push the brake pedal and decrease the vehicle velocity for safety reason. Figure 13 presents the changed longitudinal velocity of the overtaken vehicle and overtaking vehicle. Figure 14 shows the path boundary of the overtaking vehicle and this boundary is determined by the position of the overtaken vehicle and boundary of top lane and bottom lane.

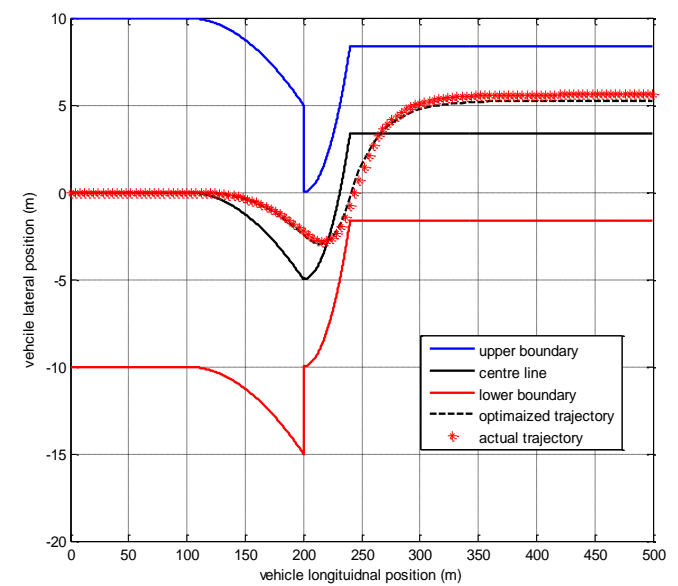

Figure 10. Vehicle actual trajectory when the surrounding traffic is considered in the third set of simulations.

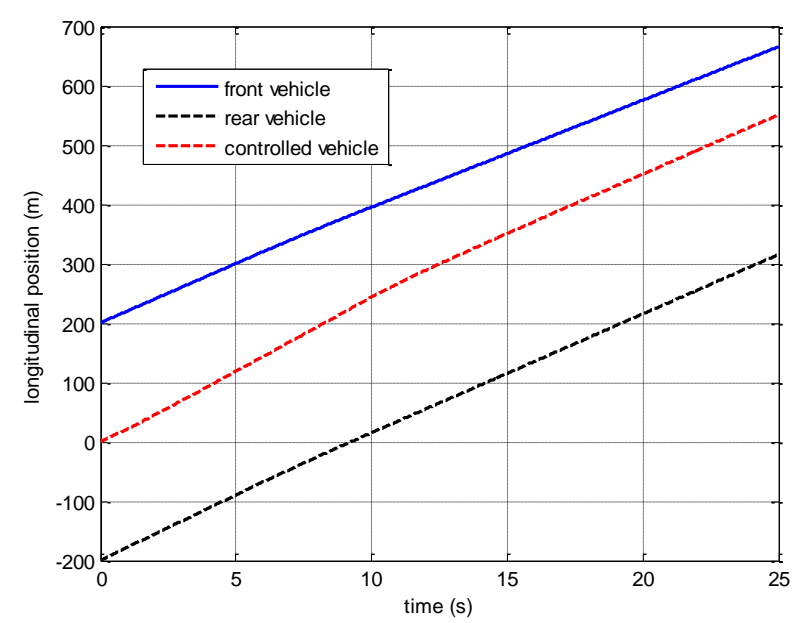

Figure 11. Vehicle longitudinal position in the body-fixed coordinate system when the surrounding traffic is considered in the third set of simulations.

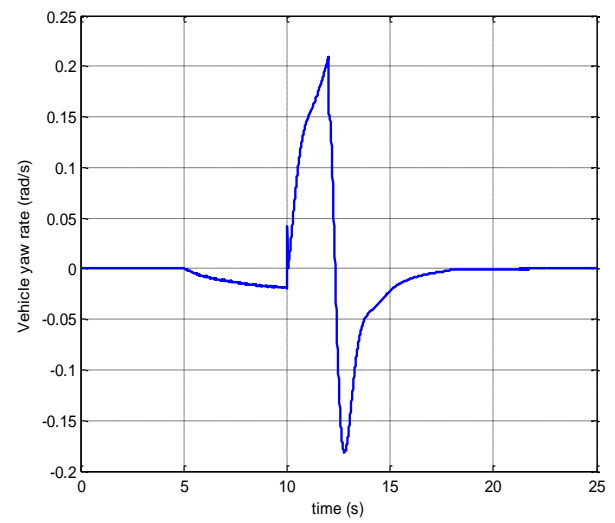

(a)

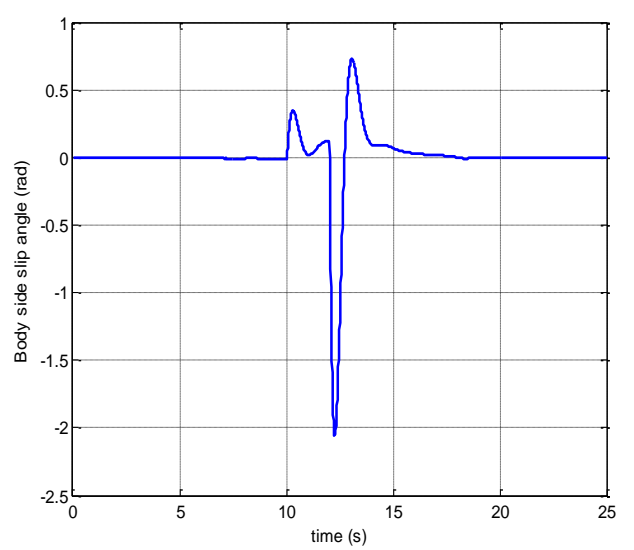

(b)

Figure 12. The actual vehicle responses (a) body slip angle (b) yaw rate in the third set of simulations.

The upper and lower boundaries (blue line and red line in Figure 14) are actually changed with the relative longitudinal position between the overtaking vehicle and overtaken vehicle. If the longitudinal position of the controlled overtaking vehicle is behind the overtaken vehicle, the overtaking vehicle is constrained within the bottom lane. If the longitudinal position 
of the overtaking vehicle is in front of the overtaken vehicle with a certain safety distance $d$, the overtaking vehicle starts to make a lane change with the following boundary condition:

$$
X_{1}>X_{2}+d
$$

where $X_{1}$ and $X_{2}$ are the longitudinal positions of the controlled overtaking vehicle and overtaken vehicle, respectively. It should be noted that the safety distance $d$ is changing with vehicle velocity in reality. In this study, however, this value is assumed as constant due to the velocity is not changed significantly.

According to Figure 14, the actual vehicle trajectory is roughly constrained by the road boundary and this proves that the proposed controller can successfully control the vehicle motion in the actual traffic condition of overtaking. In this case, $a_{0}=2, a_{v}=0.5, b_{1}=b_{2}=2000, c=20000$ are used in the optimal law (16). Figure 15 also suggests that the vehicle yaw rate and body slip angle change abruptly during the overtaking.

It is noted the actual vehicle trajectory when the desired path is strictly followed would hit the road boundary in the third and fourth set of simulations and is not presented here.

According to the four sets of simulations, the major limitations of the potential field method is the requirement of the manually tuning of the optimisation scaling factors in different scenarios, which is possibly time-consuming. In addition, the proposed method cannot handle too extreme situations, such as turning abruptly with very fast speed.

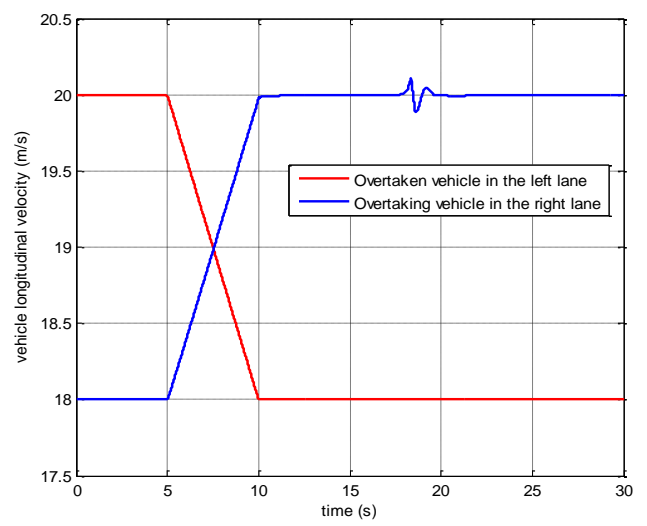

Figure 13. Vehicle longitudinal velocity of the overtaking vehicle and overtaken vehicle in the fourth set of simulations

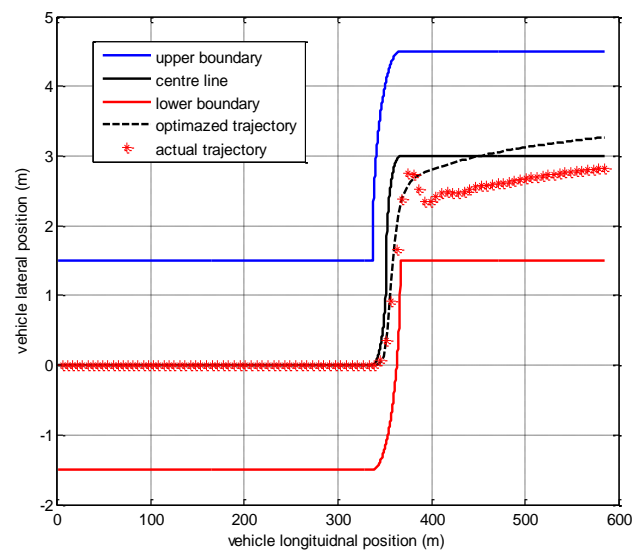

Figure 14. Vehicle actual trajectory of the controlled overtaking vehicle when the path is optimised in the fourth set of simulations

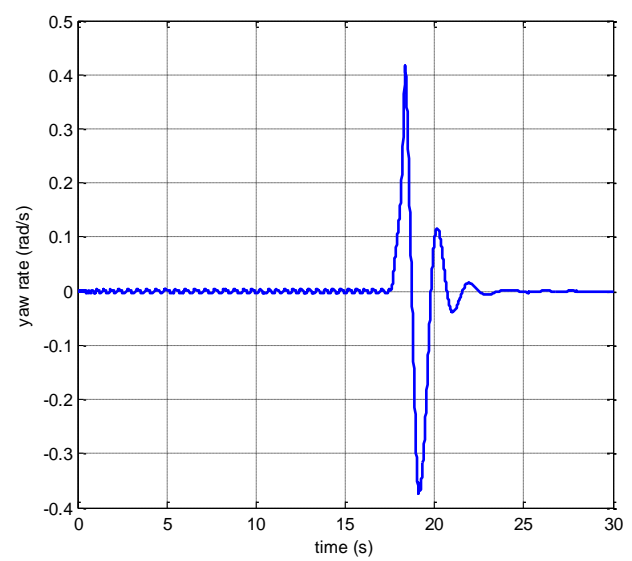

(a)

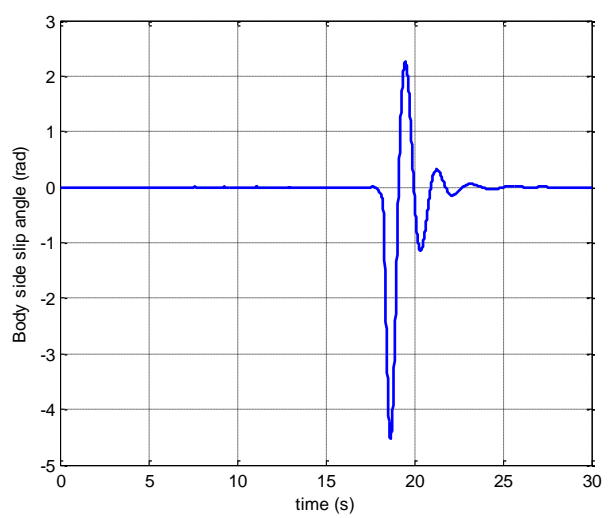

(b)

Figure 15. Vehicle (a) yaw rate and (b) body slip angle response in the fourth set of simulation

\section{CONCLUSION}

The potential field method is widely applied in the trajectory control of the mobile robotic. This study extends the potential field method into a more challenge research area - the trajectory control of the autonomous on-road vehicle that has less mobility and higher velocity compared with the mobile robot. An innovative potential field function that includes the attractive potential, the repulsive potential and the potential that minimises yaw angle change rate is proposed in this study to determine the desired yaw angle. Then according to the desired yaw angle and longitudinal velocity, the two-level vehicle trajectory controller is proposed to control the actual vehicle trajectory. The simulation results verified the suggested controller and the major findings are listed below:

1) Compared with the method that exactly tracks the road centre line, the proposed potential field method that constrains the actual vehicle trajectory in a certain road boundary has better handling and stability performance. 
2) In the narrow road boundary condition, the vehicle controlled by proposed potential field method can successfully avoid the upper and lower boundaries.

3) When the road boundary is changed in real-time due to the actual traffic condition or when the controlled vehicle tries to make the lane change and overtake other vehicles, the proposed method can still successfully control the vehicle.

This study only suggests some useful findings of the application of the potential field method to the autonomous vehicle control. In the future, the more advanced controller should be proposed to deal with more complex autonomous control problem in the macro view, such as the vehicle control in the intersection.

\section{REFERENCES}

[1] S. Hima, S. Glaser, A. Chaibet, and B. Vanholme, "Controller design for trajectory tracking of autonomous passenger vehicles," in Proc. IEEE Conf. Intell. Transp. Syst., Washington, DC, 2011, pp. 1459-1464.

[2] J. E., Naranjo, C. Gonzalez, R. Garcia, T. de Pedro, and R. E. Haber, "Power-steering control architecture for automatic driving," IEEE Transactions on Intelligent Transportation System, vol.6, no.4, pp. 406-415, 2005.

[3] J. Pérez, V. Milanés, and E. Onieva, "Cascade architecture for lateral control in autonomous vehicles", IEEE Transactions on Intelligent Transportation System, vol.12, no.1, pp. 73-82, 2011.

[4] C. Lin, J. Juang, and K. Li, "Active collision avoidance system for steering control of autonomous vehicles", IET Intelligent Transport Systems, vol.8, no.6, pp.550-557, 2014.

[5] P. Petrov, and F. Nashashibi, "Modelling and nonlinear adaptive control for autonomous vehicle overtaking", IEEE Transactions on Intelligent Transportation System, vol.15, no.4, pp.1643-1656, 2014.

[6] L. Lapierre, R. Zapata, and P. Lepinay, "Combined path-following and obstacle avoidance control of a wheeled robot", The International Journal of Robotics Research, vol.26, no.4, pp.361-375, 2007.

[7] D. Soetanto, L. Lapierre, and A. Pascoal, "Adaptive, nonsingular path following control of dynamic wheeled robot", $42^{\text {nd }}$ IEEE Conference on Decision and Control, Maui, Hawaii, USA, December, 2003, pp.1765-1770.

[8] M. A. K. Jaradat, M. H. G., Garibeh, and E. A. Feilat, "Autonomous mobile robot dynamic motion planning using hybrid fuccy potential field", Soft Comput, vol.16, pp.153-164, 2012.

[9] O. Khatib, "Real-time obstacle avoidance for manipulators and mobile robot", The International Journal of robotic Research, vol.5, no.1, pp.90-98, 1986.

[10] Z. Pan, J. Li, K. Hu, and H. Zhu, "Intelligent vehicle path planning based on improved artificial potential field method", Applied Mechanics and Materials, vol.742, pp.349-354, 2015.

[11] S. S. Ge, and Y. J. Cui, "Dynamic motion planning for mobile robots using potential field method", Autonomous Robots, vol.13, pp.207-222, 2002.

[12] H. Park, and J. C. Gerdes, "Optimal tyre force allocation for trajectory tracking with an over-actuated vehicle", 2015 IEEE Intelligent Vehicles Symposium (IV), Seoul, Korea, 2015, pp.1032-1037.

[13] R. Wang, Y. Chen, D. Feng, X. Huang, and J. Wang, "Development and performance characterization of an electric ground vehicle with independently actuated in-wheel motors", J. Power Sources, vol.196, no.8, pp.3962-3971, 2011.

[14] Y. Chen, and J. Wang, "Adaptive energy-efficient control allocation for planar motion control of over-actuated electric ground vehicles", IEEE Transactions on Control System Technology, vol.22, no.4, pp.1362-1373, 2014.

[15] J. Gu, M. Ouyang, D. Lu, J. Li, and L. Lu, "Energy efficiency optimization of electric vehicle driven by in-wheel motors", International Journal of Automotive Technology, vol.14, no.5, pp.763-772, 2013.

[16] A. Pennycott, L. De Novellis, A. Sabbatini, P. Gruber, and A. Sorniotti, "Reducing the motor power losses of a four-wheel drive, fully electric vehicle via wheel torque allocation", Proc IMechE Part D: J Automobile Engineering, vol.228, no.7, pp.830-839, 2014.
[17] M. Barth and K. Boriboonsomsin, "Energy and emission impacts of a freeway-based dynamic eco-driving system", Transportation Research Part D, vol.14, pp.400-410, 2009.

[18] Y. Saboohi and H. Farzaneh, "Model for developing an eco-driving strategy of a passenger vehicle based on the least fuel consumption", Applied Energy, vol.86, pp.1925-1932, 2009.

[19] B. Y. Li, W. H. Li, O. Kennedy, and H. P. Du, "The dynamics analysis of an omni-directional vehicle," International Journal of Automotive Technology, vol.15, no.3, pp.387-398, 2014.

[20] B. Boada, M. Boada, and V. Díaz, "Fuzzy-logic applied to yaw moment control for vehicle stability," Vehicle System Dynamics, vol.43, pp.753-770, 2005.

[21] Y. Zhao, and J. Zhang, "Yaw stability control of a four-independent-wheel drive electric vehicle", Int. J. Electric and Hybrid Vehicles, vol.2, no.1, pp.64-76, 2009.

[22] H. Dugoff, P. S. Fancher, and L. Segel, "An analysis of tire traction properties and their influence on vehicle dynamic performance", $S A E$ 700377, pp. 1219-1243, 1970.

[23] S. Glaser, B. Vanholme, S. Mammar, D. Gruyer, and L. Nouveliére, "Maneuver-based trajectory planning for highly autonomous vehicles on real road with traffic and driver interaction", IEEE Transactions on Intelligent Transportation systems, vol.11, no.3, pp.589-606, 2010.

[24] J. Wang, L. Alexander, and R. Rajamani, "Friction estimation on high-way vehicles using longitudinal measurements", ASME J. Dyn. Syst.,Meas. Control, vol.126, no.2, pp.265-275, 2004.

[25] R. Rajamani, G. Phanomchoeng, D. Piyabongkarn, and J. Y. Lew, "Algorithms for real-time estimation of individual wheel tire-road friction coefficients", IEEE/ASME Transactions on Mechatronics, vol.17, no.6, pp.1183-1195, 2012.

[26] B. Li, H. Du, and W. Li, "Comparative study of vehicle tyre-road friction coefficient estimation with a novel cost-effective method", Vehicle System Dynamics, vol.52, no.8, pp.1066-1098, 2014.

[27] Y. Chen, and J. Wang, "Design and experimental evaluations on energy efficient control allocation methods for overactuated electric vehicles: Longitudinal motion case", IEEE Transactions on Mechatronics, vol.19, no.2, pp.538-548, 2014

[28] M. Best, "Identifying tyre models directly from vehicle test data using an extended Kalman filter," Vehicle System Dynamics, vol.48, pp.171-187, 2010 .

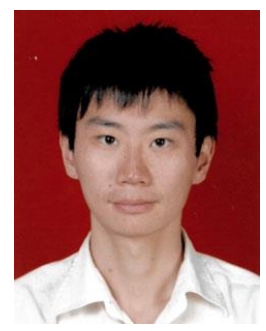

Boyuan Li received his B.E. in Mechanical Engineering and Automation from Xi'an Jiaotong University, China, in 2008. He received his Master of Engineering Practise and Master of Engineering (by research) from the School of Mechanical, Material and Mechatronics at the University of Wollongong, Australia, in 2010 and 2012, respectively. He is currently working toward his Ph.D. on vehicle dynamics and control at the School of Electrical, Computer and Telecommunications Engineering at the University of Wollongong, Australia. His research interests include vehicle

dynamics and control (for electric vehicles), four-wheel independent steering and driving vehicles, energy-efficient control, and fault-tolerant control.

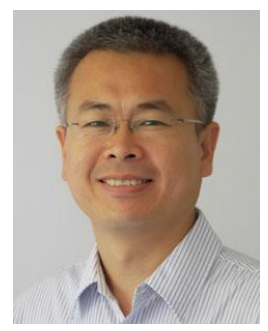

Haiping Du received his Ph.D. in mechanical design and theory from Shanghai Jiao Tong University, Shanghai, PR China, in 2002. He was Research Fellow at the University of Technology, Sydney from 2005-2009, and Post-Doctoral Research Associate at the Imperial College London (2004-2005) and 
the University of Hong Kong (2002-2003). He is currently Professor of School of Electrical, Computer and Telecommunications Engineering at the University of Wollongong. He is an Editorial Advisory Board Member of Journal of Sound and Vibration and Associate Editor of Journal of The Franklin Institute and IEEE Control Systems Society Conference.

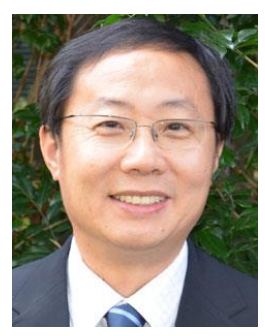

Weihua Li received his B.E. and M.E. from University of Science and Technology of China in 1992 and 1995 respectively; and his Ph.D. from Nanyang Technological University, Singapore in
2001. He was with the School of Mechanical and Production Engineering of Nanyang Technological University as a research fellow from 2001 to 2003 . He has been with the School of Mechanical, Materials and Mechatronic Engineering, University of Wollongong, as Lecturer (2003-2005), Senior Lecturer (2006-2009), Associate Professor (2010-2012), and Professor (2012-). He serves as Associate Editor or editorial board member for nine international journals. 